\title{
Competencia argumentativa: un factor clave en la formación de docentes
}

Francisco Javier Ruiz-Ortega

https://orcid.org/oooo-0003-1592-5535

Universidad de Caldas, Colombia

francisco.ruiz@ucaldas.edu.co

\section{Carmen Dussán Lubert}

https://orcid.org/00oo-0002-8093-6487 Universidad de Caldas, Colombia carmen.dussan@ucaldas.edu.co

\section{Resumen}

Este artículo aporta elementos que evidencian la necesidad de intervenir y proponer la argumentación como un contenido explícito en la formación de profesores de educación media. Para ello, se desarrolló una investigación de tipo descriptivocorrelacional, de carácter transversal, cuya población estuvo constituida por 314 estudiantes del programa de Licenciatura en Biología y Química, de la Universidad de Caldas, Colombia. Un muestreo estratificado aleatorio simple permitió determinar el tamaño de la muestra, que fue de 121 sujetos. El estudio concluye que la poca claridad para asumir la argumentación como posibilidad de aprendizaje y coconstrucción de conocimiento escolar, sumada al poco trabajo en el aula centrado en procesos argumentativos conscientes e intencionados que aporten al desarrollo de aprendizajes en profundidad, hacen indispensable en las estructuras curriculares la presencia explicita de la argumentación como objeto de enseñanza y aprendizaje.

\section{Palabras clave (Fuente: tesauro de la Unesco)}

Competencia argumentativa; desarrollo de competencias; desarrollo de habilidades; formación de profesores; planes curriculares universitarios; Universidad de Caldas, Colombia.

\footnotetext{
Recepción: 05/03/2020 | Envío a pares: 19/09/2020 | Aceptación por pares: 08/03/2021 | Aprobación: 19/04/2021 


\title{
Argument Proficiency: A Key Factor in Teacher Training
}

\begin{abstract}
The article provides elements that prove the need to take action and propose argumentation as a specific high school teacher training content. For this, we conducted a cross-sectional descriptive-correlational study, whose population comprised 314 students from the Bachelor's degree program in Biology and Chemistry at the Universidad de Caldas, Colombia. Stratified simple random sampling allowed determining the sample size, which was 121 subjects. The study concludes that a lack of clarity in accepting argumentation as a possibility for learning and co-constructing school knowledge, coupled with insufficient classroom efforts focused on conscious and intentional argumentative processes to advance learning, make it essential to include argumentation in curricular structures as a subject matter of teaching and learning.
\end{abstract}

\section{Keywords (Source: Unesco Thesaurus)}

Argumentative competence; skills development; teacher education; teacher training; university curricular plans; Universidad de Caldas, Colombia. 


\section{Competência argumentativa: um fator-chave na formação de docentes}

\section{Resumo}

Este artigo contribui com elementos que evidenciam a necessidade de intervir e propor a argumentação como conteúdo explícito na formação de professores de ensino médio. Para isso, foi desenvolvida uma pesquisa de tipo descritivo-correlacional, de caráter transversal, cuja população esteve constituída de 314 estudantes do programa de licenciatura em Biologia e Química, da Universidad de Caldas, Colômbia. Uma amostragem aleatória estratificada simples permitiu determinar o tamanho da amostra, que foi de 121 sujeitos. Neste estudo, conclui-se que a pouca clareza para assumir a argumentação como possibilidade de aprendizagem e coconstrução de conhecimento escolar, somada ao escasso trabalho na sala de aula focado em processos argumentativos conscientes e intencionados que contribuam para desenvolver aprendizagens em profundidade tornam indispensável a presença explícita da argumentação como objeto de ensino e aprendizagem nas estruturas curriculares.

\section{Palavras-chave (Fonte: tesauro da Unesco)}

Competência argumentativa; desenvolvimento de competências; desenvolvimento de habilidades; formação de professores; planos curriculares universitários; Universidad de Caldas, Colômbia. 
Bajo la premisa de que "los docentes son agentes de cambio de las sociedades" (Landazábal y Gamboa, 2018, p. 27), se considera que una de las responsabilidades de las instituciones formadoras de docentes tiene que ver con el desarrollo de la participación, entendida como competencia necesaria e indispensable para las prácticas cotidianas de los futuros docentes. En este sentido, es importante reconocer los esfuerzos de muchas instituciones educativas que intentan incorporar en los procesos de formación docente escenarios de reflexión y posible transformación de sus propias prácticas (Cochran y Lytle, 2003; Tezanos, 2013). No obstante, es fácil encontrar estructuras curriculares rígidas, descontextualizadas (Ruiz et al., 2018; Ruiz, 2016; Mellado, 2010) y centradas en el contenido (Echeverría, 2010), que caracterizan los procesos de formación docente y limitan este propósito. Características no solo de los programas de formación de docentes, sino también de los procesos de formación continua, donde prima la racionalidad técnica, que asume al docente como ejecutor de modelos o métodos generados en procesos de investigación ajenos a sus propios contextos (Mellado, 2001).

Ahora bien, lograr desarrollar en los futuros docentes la participación, como competencia necesaria para el cambio social, exige innegablemente cambiar los procesos de comunicación de los sujetos. Cambio que pasa por el desarrollo de la argumentación como proceso comunicacional, dialógico y dialéctico. Para Mórtimer y Machado, "la toma de consciencia y participación de los estudiantes (futuros docentes, en nuestro caso) en la resolución de conflictos depende no solo de la selección de unas estrategias adecuadas, sino, sobre todo, del discurso construido en torno a la actividad" (2001, citados por Archila, 2014, p. 62).

La argumentación en ciencias es un proceso comunicativo con carácter dialógico, dialéctico, situado y multimodal (Ruiz y Ocampo, 2019; Archila, 2014), que permite a los futuros docentes, entre otras cosas, construir aprendizajes con sentido y significado para sus desempeños; exponer sus pensamientos y modelos explicativos sobre los fenómenos estudiados en el aula (naturales, sociales, educativos); jalonar el desarrollo de actitudes y valores; participar de manera razonada en la toma de decisiones y resolver problemas auténticos y relevantes para su propio proceso formativo y profesional (Jiménez, 1998).

Asumir el reto de su desarrollo en el aula de ciencias exige, entre otras cosas, la contextualización de los conocimientos que se comunican en ella, contextualización que, como proceso, debe caracterizarse por ser relevante, realista, significativa y por permitir la transferibilidad del saber a contextos diferentes de aquellos diseñados en los espacios educativos (Sanmartí, 2019).

De igual manera, es importante enfatizar en que una relación afectiva entre los implicados en los debates podría ayudar a lograr el objetivo de un proceso argumentativo: el consenso, el convencimiento, la persuasión o la coconstrucción de conocimiento (Paglieri y Castelfranchi, 2010); por ello, no es posible separar los procesos argumentativos de aspectos emocionales y metacognitivos, que condicionan la participación y el rol de cada sujeto en los debates que se promuevan en el aula.

En relación con lo emocional, cuando se participa en un debate, en un proceso argumentativo, existen riesgos o "peligros" que conllevan cierta responsabilidad personal; esto condiciona, en determinados contextos y ciertas situaciones, la toma de decisiones que definen la participación o no en dichos debates (Santibáñez, 2014).

En relación con lo metacognitivo, si pretendemos desarrollar procesos de aprendizaje en profundidad y aportar al desarrollo de pensadores críticos, será necesario que los estudiantes aprendan a ser conscientes de sus propias dificultades y de la manera cómo planean, monitorean y evalúan sus propios procesos de aprendizaje. Es decir, convertir los escenarios evaluativos en verdaderos escenarios de aprendizaje. 
Pese a este evidente llamado a incorporar la argumentación como una competencia relevante en el proceso formativo de cada estudiante y, en nuestro caso, de aquellas personas que se forman como futuros docentes, la literatura revisada indica que la argumentación, como contenido explícito en la formación de docentes, es una tarea pendiente y, peor aún, que los futuros docentes no están preparados para enseñar a argumentar, lo cual es consecuencia lógica de las deficiencias y vacíos en los procesos de formación docente recibidos en las instituciones educativas (Ruiz et al., 2018; Lourenço, Abib y Murillo, 2016; Cutrera y Stipcich, 2015; Archila, 2014; Muller y Perret, 2009; Zohar, 2007; Stipcich, 2005; Stipcich et al., 2006; Vianna y Carvalho, 2000).

En armonía con los planteamientos de Archila (2016) y tratando de dar respuesta a ese gran reto que presentan los programas de formación de docentes en el sentido de "aportar a la formación de docentes participativos en los cambios de la sociedad", se despliegan otros interrogantes que el presente artículo pretende explorar: ¿qué es argumentar en ciencias?, ¿para qué promover la argumentación en ciencia escolar?, ¿cómo fomentar la argumentación en clase de ciencias?, ¿cuál es la percepción, como futuro docente, del reto de enseñar a argumentar en ciencias?, ¿qué criterios en relación con el docente, el estudiante y el contenido disciplinar se consideran necesarios para facilitar el desarrollo de la argumentación en ciencias?, ¿cuáles son las prácticas más comunes que se vivencian en las aulas de clase y cómo estas aportan al desarrollo de la argumentación en ciencias? y ¿cuáles son los juicios de orden metacognitivo que, como futuro docente, se tiene sobre la enseñanza de la argumentación en ciencias?

En definitiva, este artículo pretende, con base en los resultados obtenidos en un programa de formación de docentes (licenciatura en Biología y Química de la Universidad de Caldas)', aportar a

$1 \quad$ El programa de Licenciatura en Biología y Química, ahora denominado Licenciatura en Ciencias Naturales, dadas las la reflexión sobre por qué se considera necesario intervenir y proponer la argumentación como un contenido explícito en la formación de licenciados en este campo de conocimiento. Para ello, se parte del conocimiento y experiencia de los futuros docentes de la licenciatura en Biología y Química, para ver lo que piensan sobre la argumentación en ciencias, junto con el qué, el cómo y el para qué de esta competencia, y para identifica de qué modo su vivencia en el aula los autoriza a cuestionarse qué tan lejos se está de ser mediadores del desarrollo de esta competencia en el aula y de aportar con la enseñanza a dicho objetivo.

\section{Metodología}

La investigación es de tipo descriptivo-correlacional y de carácter transversal (Hernández, Fernández y Baptista, 2010). La población estuvo constituida por todos los estudiantes matriculados durante el segundo semestre de 2018 en el programa de Licenciatura en Biología y Ouímica de la Universidad de Caldas (314). Un muestreo estratificado aleatorio simple para proporciones, con un error del $7 \%$, permitió determinar el tamaño de la muestra (121 estudiantes), tamaño que se incrementó a 133, pues algunos estudiantes solicitaron de manera voluntaria participar de la investigación y firmar el consentimiento informado. El único criterio de

exigencias de la política educativa colombiana, tiene una amplia trayectoria en la formación de licenciados, con más de 40 años de historia en la ciudad de Manizales, la región y el país. En uno de los documentos maestros que sintetiza la trayectoria, el impacto y los proyectos a mediano y largo plazo se muestra cómo este programa propone como objetivos fundamentales en la formación de docentes: "formar educadores calificados de las Ciencias Naturales, capaces de abordar reflexiones sobre aspectos curriculares, didácticos y de aprendizaje, y de proyectarse a la formación post-graduada en estas temáticas"; "contribuir en la generación de alternativas metodológicas para la enseñanza de las Ciencias Naturales"; "impartir una sólida formación en Ciencias Naturales, necesaria para acceder a la formación post-graduada en estas disciplinas o para integrarse en equipos de investigación interdisciplinarios"; y "generar semilleros de profesionales con la conciencia de la importancia de las ciencias básicas en la construcción de las disciplinas aplicadas" (Documento Maestro de acuerdo con el Decreto 1075/2015 del Ministerio de Educación Nacional Colombiano). 
inclusión fue estar matriculado durante dicho semestre en la Licenciatura en Biología y Química de la Universidad de Caldas.

Para la obtención de la información se aplicó un cuestionario, que ha sido validado mediante juicio de expertos y prueba piloto. El cuestionario, en las primeras 12 preguntas, indaga por el semestre, el género de los estudiantes, el concepto de la argumentación, las actividades necesarias para su desarrollo en el aula, y por aspectos relacionados con sus emociones ante el reto de asumir la tarea de desarrollar la argumentación en el aula de ciencias. Las últimas tres preguntas, de naturaleza cualitativa nominal, buscaron identificar si los estudiantes lograban identificar, en un texto argumentativo, ${ }^{2}$ sus elementos estructurales.

2 El texto en el cual debían identificar la tesis, los datos y la justificación fue: "La acreditación de las Instituciones de Educación Superior la define el Consejo Nacional de Acreditación (CNA) como 'el acto por el cual el Estado adopta y hace público el reconocimiento que los pares académicos hacen de la comprobación que efectúa una institución sobre la calidad de sus programas académicos, su organización y funcionamiento y el cumplimiento de su función social' (https://www. cna.gov.co/1741/article-187231.html). Nuestra institución obtuvo en el año 2018 y, por ocho años, la renovación de la Acreditación de Alta Calidad. Es un logro que sin duda se debe a que nuestra institución ha mejorado muchos aspectos, algunos de ellos; en el 2012 cuando se obtuvo por primera vez la re-acreditación, se tenían 77 docentes con formación doctoral y, en el 2017 (primer semestre) este número se incrementó a 142. A nivel de deserción estudiantil, nuestra universidad, para el año 2016, mostró una deserción inferior a la que se presenta en el conjunto en las instituciones de educación superior colombianas y las universidades en particular (Informe de autoevaluación institucional, p. 26). También se evidenció, en el proceso de evaluación de pares, que nuestra universidad cuenta con programas de flexibilización curricular como: Sistema de equivalencias, reconocimientos y participación académica acreditable; Programas en doble titulación; Doble programa en pregrado; entre otros. Finalmente, otro de los grandes hallazgos en dicho proceso de evaluación, fue que además de los docentes investigadores reconocidos en Colciencias (109 para el año 2016, según fuente de Colciencias), nuestra institución fomenta el desarrollo de procesos investigativos con sus estudiantes, prueba de ello son los 128 semilleros de investigación (108 más que los registrados en la oficina de investigaciones para el año 2012) con un total de 264 estudiantes participantes en ellos. Ahora, es ésta la información que apoya la obtención de la re-acreditación, ya que si una institución, además de los avances en otros factores que evalúa el CNA, evidencia su preocupación y hace efectivas sus estrategias para formar a sus docentes en los niveles académicos más altos; promueve estrategias de retención estudiantil para evitar

\section{Resultados}

Se describen las variables utilizando el porcentaje de estudiantes que respondió a cada pregunta. Posteriormente, se determina, mediante la prueba Chi-cuadrado de Pearson (Sprent y Smeeton, 2007), si existía independencia entre las categorías de cada pregunta, el género del estudiante y el semestre que cursa (bajo: 60 o menos créditos; medio: entre 61 y 120 créditos; alto: mayor de 120 créditos). Los análisis estadísticos fueron realizados utilizando el software XLSTAT 2014. La muestra de 133 personas estuvo constituida por 77 mujeres (57,9\%) y 56 hombres (42,1\%), discriminados por semestre así: $60,2 \%$ de primero a cuarto, $22,6 \%$ de quinto o sexto y $17,3 \%$ de séptimo, octavo, noveno o décimo.

\section{Concepto de los estudiantes sobre lo que significa argumentar en clase de ciencias}

La Tabla 2 muestra que el $84,2 \%$ de los estudiantes tiende a calificar con los valores más altos (4 o 5) todos los conceptos, en particular el segundo: "La argumentación es un proceso social, dialógico y dialéctico que permite la transformación y evolución del conocimiento científico y escolar. Es un proceso enmarcado en contextos particulares, bidireccional y rico en aspectos motivacionales, reflexivos, autorreguladores, y orientado a varios propósitos", y cuarto "La argumentación es un proceso lógico, de expresión de contenidos autónomos, que da importancia a la estructura lógica de los argumentos expuestos, desde los cuales la inferencia lógica sobre las premisas permite la construcción de conclusiones verdaderas". No se encontró dependencia significativa entre las calificaciones dadas entre hombres y mujeres para cada una de las preguntas (valores $P$ mayores de 0,139) ni entre semestres (valores P mayores de 0,193$)$.

\footnotetext{
frustraciones individuales, familiares y sociales y, también, mediante sus procesos de investigación se hace visible a nivel político, educativo, técnico y tecnológico, dando cumplimiento a su función social, demuestra con suficiencia el estar dentro de los estándares de calidad exigidos para la obtención de este certificado".
} 
Tabla 1. Preguntas realizadas en la investigación

\begin{tabular}{|c|c|}
\hline Pregunta & Ítems \\
\hline $\begin{array}{l}\text { Conceptos sobre lo que } \\
\text { supone es argumentar } \\
\text { en clase de ciencias. }\end{array}$ & $\begin{array}{l}\text { a. La argumentación es un acto comunicativo, de interacción social, presente en los contextos } \\
\text { cotidianos de los sujetos. Un acto ligado a los razonamientos informales y retóricos. } \\
\text { b. La argumentación es un proceso social, dialógico y dialéctico que permite la } \\
\text { transformación y evolución del conocimiento científico y escolar. Es un proceso enmarcado } \\
\text { en contextos particulares, bidireccional y rico en aspectos motivacionales, reflexivos, } \\
\text { autorreguladores, y orientado a varios propósitos. } \\
\text { c. La argumentación es un proceso comunicativo cotidiano, contextualizado, que se } \\
\text { caracteriza por su naturaleza social, crítica, dialéctica y pragmática, en donde las } \\
\text { diferencias se asumen como opciones que enriquecen la construcción de las soluciones. } \\
\text { d. La argumentación es un proceso lógico, de expresión de contenidos autónomos, que } \\
\text { da importancia a la estructura lógica de los argumentos expuestos, desde los cuales la } \\
\text { inferencia lógica sobre las premisas permite la construcción de conclusiones verdaderas. }\end{array}$ \\
\hline $\begin{array}{c}\text { Valoración de los } \\
\text { objetivos vinculados a } \\
\text { la argumentación en } \\
\text { ciencias. }\end{array}$ & $\begin{array}{l}\text { a. Se centra en la reestructuración del conocimiento para aportar a la resolución de problemas } \\
\text { contextuales. } \\
\text { b. Se centra en el convencimiento y la fortaleza que debe tener la estructura argumentativa } \\
\text { y su forma lógica. Es fundamental la aplicación de patrones de inferencia, es decir, deducir } \\
\text { desde las premisas la conclusión. } \\
\text { c. Se centra en persuadir al auditorio o a un interlocutor sobre ciertas creencias o disposiciones } \\
\text { o en provocar en ellos determinadas actuaciones o reacciones, para lograr su adhesión. } \\
\text { d. Se centra en la resolución de las diferencias de opinión. }\end{array}$ \\
\hline $\begin{array}{c}\text { Valoración de la } \\
\text { presencia de algunas } \\
\text { actividades en las } \\
\text { clases. }\end{array}$ & $\begin{array}{l}\text { a. Exposiciones. } \\
\text { b. Debates. } \\
\text { c. Resolución de ejercicios matemáticos, físicos o químicos. } \\
\text { d. Resolución de controversias sociocientíficos. } \\
\text { e. Análisis de casos. } \\
\text { f. Pruebas escritas. } \\
\text { g. Elaboración de ensayos. } \\
\text { h. Trabajos individuales. } \\
\text { i. Trabajos grupales. }\end{array}$ \\
\hline $\begin{array}{l}\text { Valoración de algunas } \\
\text { actividades, de acuerdo } \\
\text { con la pertinencia } \\
\text { para desarrollar la } \\
\text { argumentación en el } \\
\text { aula de ciencias. }\end{array}$ & $\begin{array}{l}\text { a. Exposiciones. } \\
\text { b. Debates. } \\
\text { c. Resolución de ejercicios matemáticos, físicos o químicos. } \\
\text { d. Resolución de controversias sociocientíficos. } \\
\text { e. Análisis de casos. } \\
\text { f. Pruebas escritas. } \\
\text { g. Elaboración de ensayos. } \\
\text { h. Trabajos individuales. } \\
\text { i. Trabajos grupales. }\end{array}$ \\
\hline $\begin{array}{l}\text { Si el reto es desarrollar } \\
\text { la argumentación en } \\
\text { el aula, valoración de } \\
\text { la importancia que } \\
\text { debe darse a algunos } \\
\text { aspectos relacionados } \\
\text { con el docente. }\end{array}$ & $\begin{array}{l}\text { a. Saber contextualizar los contenidos. } \\
\text { b. Saber la disciplina que enseña. } \\
\text { c. Saber motivar a sus estudiantes. } \\
\text { d. Saber evaluar la argumentación. } \\
\text { e. Saber preguntar. }\end{array}$ \\
\hline
\end{tabular}




\begin{tabular}{|c|c|}
\hline Pregunta & Ítems \\
\hline $\begin{array}{l}\text { Si el reto es desarrollar } \\
\text { la argumentación en } \\
\text { el aula, valoración de } \\
\text { la importancia que } \\
\text { debe darse a algunos } \\
\text { aspectos relacionados } \\
\text { con el estudiante. }\end{array}$ & $\begin{array}{l}\text { a. Tener conocimientos disciplinares. } \\
\text { b. Tener un conocimiento general, pero también el detalle de lo que se discute. } \\
\text { c. Tener actitud y motivación hacia las ciencias y sus implicaciones. } \\
\text { d. Saber escuchar al otro en una discusión. } \\
\text { e. Saber identificar en los argumentos los aspectos que generan controversia. }\end{array}$ \\
\hline $\begin{array}{l}\text { Si el reto es desarrollar } \\
\text { la argumentación en } \\
\text { el aula, valoración de } \\
\text { la importancia que } \\
\text { debe darse a algunos } \\
\text { aspectos relacionados } \\
\text { con el contenido. }\end{array}$ & $\begin{array}{l}\text { a. Estar contextualizados. } \\
\text { b. Ser problémicos. }\end{array}$ \\
\hline $\begin{array}{l}\text { Si te pidieran ubicarte } \\
\text { en el rol de docente, } \\
\text { indica para cada pareja } \\
\text { de adjetivos la posición } \\
\text { que mejor expresa tu } \\
\text { percepción de promover } \\
\text { la argumentación en } \\
\text { clase de ciencias. }\end{array}$ & $\begin{array}{l}\text { a. Participativa/Pasiva. } \\
\text { b. Estresante/Relajada. } \\
\text { c. Compleja/Simple. } \\
\text { d. Entretenida/Aburrida. } \\
\text { e. Optimista/Pesimista. } \\
\text { f. Superficial/Profunda. } \\
\text { g. Positiva/Negativa. } \\
\text { h. Teórica/Práctica. } \\
\text { i. Relevante/Secundaria. }\end{array}$ \\
\hline $\begin{array}{c}\text { Si te pidieran ubicarte } \\
\text { en el rol de docente de } \\
\text { ciencias naturales, lee } \\
\text { cuidadosamente cada } \\
\text { declaración y elije la } \\
\text { opción que consideres } \\
\text { mejor. }\end{array}$ & $\begin{array}{l}\text { a. Sé lo necesario sobre la argumentación para promoverla en el aula. } \\
\text { b. Podría explicar con mis palabras lo que es la argumentación en ciencias. } \\
\text { c. Conozco mis debilidades y fortalezas en lo que significa argumentar en ciencias y su } \\
\text { enseñanza en el aula. } \\
\text { d. Sé cómo promover la argumentación en el aula. } \\
\text { e. Sé lo que se espera lograr cuando se enseña a argumentar en el aula. } \\
\text { f. Sé cuándo un estudiante argumenta en clase de ciencias. } \\
\text { g. Sé identificar los datos de un argumento. } \\
\text { h. Sé identificar los fundamentos teóricos que sustentan los argumentos. } \\
\text { i. Pienso en lo que necesito saber antes de iniciar la ejecución de la tarea. } \\
\text { j. Tengo un propósito claro para la ejecución de la tarea. }\end{array}$ \\
\hline
\end{tabular}




\begin{tabular}{|c|c|}
\hline Pregunta & Ítems \\
\hline $\begin{array}{c}\text { Si te pidieran ubicarte } \\
\text { en el rol de docente de } \\
\text { ciencias naturales, lee } \\
\text { cuidadosamente cada } \\
\text { declaración y elije la } \\
\text { opción que consideres } \\
\text { mejor. }\end{array}$ & $\begin{array}{l}\text { k. Analizo la utilidad de las posibles estrategias para promover la argumentación en ciencias. } \\
\text { l. Sé cómo abordar los contenidos para facilitar la argumentación en ciencias. } \\
\text { m. Estoy seguro de lo que creo saber sobre la argumentación en ciencias y su enseñanza. } \\
\text { n. Sé identificar las justificaciones en un argumento. } \\
\text { o. Pienso que lo que sé sobre la argumentación en ciencias es la perspectiva que debe } \\
\text { promoverse en el aula. } \\
\text { p. Pienso que no tendré dificultades para lograr promover la argumentación en ciencias en el } \\
\text { aula. } \\
\text { q. Analizo la utilidad, para mi desempeño profesional, de emprender este reto e intentar } \\
\text { lograrlo. } \\
\text { r. Soy consciente de que el interés que tengo para el desarrollo de la tarea facilita el logro del } \\
\text { objetivo propuesto. } \\
\text { s. Creo que con lo que sé sobre la argumentación en ciencias y su enseñanza es suficiente } \\
\text { para lograrla en el aula. } \\
\text { t. Sé identificar la tesis central en un argumento. }\end{array}$ \\
\hline \multicolumn{2}{|c|}{ Del texto anterior, cuál es el fragmento que expresa los datos. } \\
\hline \multicolumn{2}{|c|}{ Del texto anterior, cuál es el fragmento que expresa la tesis central. } \\
\hline
\end{tabular}

Fuente: elaboración propia.

Tabla 2. Valoración de los estudiantes de lo que supone argumentar en clase de ciencias (\%)

\begin{tabular}{|c|c|c|c|c|c|}
\hline \multirow{2}{*}{ Concepto } & \multicolumn{5}{|c|}{ Valoración (\%) } \\
\hline & 1 & 2 & 3 & 4 & 5 \\
\hline $\begin{array}{l}\text { La argumentación es un acto comunicativo, de interacción social, presente en los } \\
\text { contextos cotidianos de los sujetos. Un acto ligado a los razonamientos informales y } \\
\text { retóricos. }\end{array}$ & 5,3 & 10,5 & 18,0 & 41,4 & 24,8 \\
\hline $\begin{array}{l}\text { La argumentación es un proceso social, dialógico y dialéctico que permite la } \\
\text { transformación y evolución del conocimiento científico y escolar. Es un proceso } \\
\text { enmarcado en contextos particulares, bidireccional y rico en aspectos motivacionales, } \\
\text { reflexivos, autorreguladores, y orientado a varios propósitos. }\end{array}$ & 1,5 & 3,0 & 11,3 & 30,1 & 54,1 \\
\hline $\begin{array}{l}\text { La argumentación es un proceso comunicativo cotidiano, contextualizado, que } \\
\text { se caracteriza por su naturaleza social, crítica, dialéctica y pragmática, en donde } \\
\text { las diferencias se asumen como opciones que enriquecen la construcción de las } \\
\text { soluciones. }\end{array}$ & 0,8 & 4,5 & 21,8 & 35,3 & 37,6 \\
\hline $\begin{array}{l}\text { La argumentación es un proceso lógico, de expresión de contenidos autónomos, } \\
\text { que da importancia a la estructura lógica de los argumentos expuestos, desde los } \\
\text { cuales la inferencia lógica sobre las premisas permite la construcción de conclusiones } \\
\text { verdaderas. }\end{array}$ & 3,8 & 7,5 & 15,0 & 29,3 & 44,4 \\
\hline
\end{tabular}

Fuente: elaboración propia. 
La segunda pregunta indagó por el objetivo de la argumentación en ciencias. En la Tabla 3 se observa dispersión en las valoraciones realizadas por los estudiantes y poca claridad en este aspecto, pues se esperaría que el propósito de la argumentación en el campo de la enseñanza y aprendizaje de las ciencias se proyecte hacia la reestructuración de conocimiento. Adicionalmente, en la pregunta "Se centra en la resolución de las diferencias de opinión", las estudiantes tienden a calificar alto y sus compañeros a calificar bajo (valor $\mathrm{P}=0,024$ ); por su parte, los estudiantes de primeros semestres tienden a calificar más alto que los de últimos semestres (valores $\mathrm{P}$ de 0,024 y 0,008, respectivamente). En las demás preguntas no hubo dependencia estadística por género o semestre (valores P mayores de 0,242).

Al sumar las valoraciones más altas en las Tablas 4 y 5 , se encuentra que $91,7 \%, 78,2 \%$ y $72,2 \%$ de los estudiantes consideran que la resolución de problemas, las pruebas escritas y los trabajos grupales, son las actividades más presentes en las clases, mientras que la resolución de controversias sociocientíficas, los debates y la elaboración de ensayos no constituyen una tercera parte de tales las actividades. Y, al analizar las actividades que consideran más pertinentes para desarrollar la argumentación, aparecen los debates, trabajos grupales y resolución de controversias sociocientíficas como las más necesarias. O sea, que únicamente hay coincidencia en los trabajos grupales, como una actividad necesaria y que se esté realizando.

Cuando se analiza al docente en cuanto al reto de desarrollar argumentación en el aula, cuando menos el 78,9\% de los estudiantes considera que todos los aspectos evaluados son importantes o muy importantes, en particular, saber la disciplina que se enseña (Tabla 6). Entre el semestre y el aspecto evaluado se encontró independencia estadística (valores $\mathrm{P}$ mayores de 0,222), así mismo entre este último y el género del estudiante (valores $P$ de 0,366 o mayores), con excepción del caso en que para las mujeres es más importante que el docente sepa evaluar la argumentación que para sus compañeros hombres (valor $\mathrm{P}=0,028$ ).

En este mismo sentido, cuando se analiza al estudiante en cuanto al reto de desarrollar argumentación en el aula, el 93,2\% de los estudiantes evalúan como importante o muy importante el saber identificar en los argumentos los aspectos que generan controversia; en este mismo nivel de importancia, el 92,5\% y el 91,7\% valoran el saber escuchar al otro en una discusión y tener un conocimiento general, pero también el detalle de lo que se discute, respectivamente (Tabla 7). Nuevamente se encontró independencia estadística (valores $\mathrm{P}$ mayores de 0,266) entre estos aspectos, el género y el semestre.

No se encontró dependencia en las respuestas dadas por hombres y mujeres o por estudiantes de diferentes semestres en cuanto al contenido para desarrollar la argumentación en el aula (valores P mayores de 0,554). Además, el 96,2\% de los estudiantes consideraron importante o muy importante el estar contextualizados, pero solo el $61,7 \%$ consideraron importante o muy importante el ser problémicos.

Cuando se solicitó al estudiante ubicarse en el rol del docente para promover la argumentación en clase de ciencias, valorando dos adjetivos (1 se acerca más al adjetivo de la izquierda, 5 se acerca más al de la derecha), se encontró que prefieren clases participativas (52,6\%), relajadas $(37,6 \%)$, entretenidas $(63,2 \%)$, optimistas (53,4\%), profundas $(44,4 \%)$, positivas $(52,6 \%)$, prácticas $(29,3 \%)$ y relevantes $(43,6 \%)$. Mientras que en la complejidad se ubican en un término medio (Tabla 8); así mismo, se encontró que en general todos los estudiantes prefieren las clases prácticas, pero los de semestres intermedios prefieren las teóricas (valor $P=0,036$ ). El género no es dependiente de ninguna de las respuestas mostradas en la Tabla 8 (valores P de 0,127 o mayores). 
Tabla 3. Valoración dada por los estudiantes a los objetivos vinculados a la argumentación en ciencias (\%)

\begin{tabular}{|c|c|c|c|c|c|}
\hline \multirow{2}{*}{ Concepto } & \multicolumn{5}{|c|}{ Valoración (\%) } \\
\hline & 1 & 2 & 3 & 4 & 5 \\
\hline $\begin{array}{l}\text { Se centra en la reestructuración del conocimiento para aportar a la resolución de } \\
\text { problemas contextuales. }\end{array}$ & 2,3 & 6,8 & 30,8 & 31,6 & 28,6 \\
\hline $\begin{array}{l}\text { Se centra en el convencimiento y la fortaleza que deben tener la estructura } \\
\text { argumentativa y su forma lógica. Es fundamental la aplicación de patrones de } \\
\text { inferencia, es decir, deducir de las premisas la conclusión. }\end{array}$ & 1,5 & 4,5 & 18,0 & 45,1 & 30,8 \\
\hline $\begin{array}{l}\text { Se centra en persuadir en el auditorio o en un interlocutor ciertas creencias o } \\
\text { disposiciones o a provocar en ellos determinadas actuaciones o reacciones, para } \\
\text { lograr su adhesión. }\end{array}$ & 12,0 & 22,6 & 33,1 & 21,1 & 9,8 \\
\hline Se centra en la resolución de las diferencias de opinión. & 7,5 & 15,0 & 24,8 & 26,3 & 19,5 \\
\hline
\end{tabular}

Fuente: elaboración propia.

Tabla 4. Presencia de algunas actividades en clase (\%)

\begin{tabular}{|l|c|c|c|c|c|c|}
\hline \multicolumn{1}{c|}{ Actividad } & \multicolumn{5}{c|}{ Valoración (\%) } \\
\cline { 2 - 8 } & $\mathbf{1}$ & $\mathbf{2}$ & $\mathbf{3}$ & $\mathbf{4}$ & $\mathbf{5}$ & No sabe \\
\hline Exposiciones. & 6,8 & 16,5 & 28,6 & 27,8 & 19,5 & 0,8 \\
\hline Debates. & 24,1 & 24,8 & 18,8 & 21,8 & 9,8 & 0,8 \\
\hline Resolución de ejercicios matemáticos, físicos o químicos. & 0,8 & 1,5 & 5,3 & 21,8 & 69,9 & 0,8 \\
\hline Resolución de controversias sociocientíficas. & 12,8 & 20,3 & 33,8 & 19,5 & 9,0 & 4,5 \\
\hline Análisis de casos. & 6,8 & 9,8 & 26,3 & 32,3 & 22,6 & 2,3 \\
\hline Pruebas escritas. & 3,0 & 3,0 & 15,0 & 24,8 & 53,4 & 0,8 \\
\hline Elaboración de ensayos. & 13,5 & 18,8 & 32,3 & 24,1 & 9,0 & 2,3 \\
\hline Trabajos individuales. & 1,5 & 8,3 & 18,8 & 36,1 & 33,8 & 1,5 \\
\hline Trabajos grupales. & 3,8 & 3,8 & 18,8 & 39,8 & 32,3 & 1,5 \\
\hline
\end{tabular}

Fuente: elaboración propia.

Tabla 5. Valoración por el estudiante de la pertinencia de algunas actividades en clase para desarrollar la argumentación (\%)

\begin{tabular}{|l|c|c|c|c|c|c|}
\hline \multicolumn{1}{|c|}{ Actividad } & \multicolumn{5}{c|}{ Valoración (\%) } \\
\cline { 2 - 9 } & $\mathbf{1}$ & $\mathbf{2}$ & $\mathbf{3}$ & $\mathbf{4}$ & $\mathbf{5}$ & No sabe \\
\hline Exposiciones. & 3,8 & 8,3 & 27,1 & 29,3 & 30,8 & 0,8 \\
\hline Debates. & 3,0 & 5,3 & 16,5 & 23,3 & 51,9 & 0,0 \\
\hline Resolución de problemas matemáticos, físicos o químicos. & 0,8 & 9,0 & 24,1 & 33,1 & 32,3 & 0,8 \\
\hline Resolución de controversias sociocientíficas. & 2,3 & 11,3 & 14,3 & 36,1 & 33,1 & 3,0 \\
\hline Análisis de casos. & 0,0 & 5,3 & 13,5 & 35,3 & 45,1 & 0,8 \\
\hline Pruebas escritas. & 9,0 & 16,5 & 21,1 & 33,1 & 19,5 & 0,8 \\
\hline Elaboración de ensayos. & 4,5 & 12,0 & 22,6 & 30,8 & 29,3 & 0,8 \\
\hline Trabajos individuales. & 8,3 & 14,3 & 27,1 & 25,6 & 24,1 & 0,8 \\
\hline Trabajos grupales. & 3,0 & 7,5 & 20,3 & 40,6 & 27,8 & 0,8 \\
\hline
\end{tabular}

Fuente: elaboración propia. 
Tabla 6. Importancia que debe darse a aspectos relacionados con el docente (\%)

\begin{tabular}{|l|c|c|c|c|c|}
\hline \multirow{2}{*}{ Aspecto } & \multicolumn{5}{c|}{ Valoración* (\%) } \\
\cline { 2 - 7 } & MPI & PI & Mel & I & MI \\
\hline Saber contextualizar los contenidos. & 1,5 & 2,3 & 2,3 & 18,8 & 75,2 \\
\hline Saber la disciplina que enseña. & 0,8 & 0,8 & 2,3 & 15,8 & 80,5 \\
\hline Saber motivar a sus estudiantes. & 1,5 & 1,5 & 5,3 & 21,1 & 70,7 \\
\hline Saber evaluar la argumentación. & 1,5 & 0,8 & 7,5 & 25,6 & 64,7 \\
\hline Saber preguntar. & 1,5 & 2,3 & 3,0 & 2,8 & 80,5 \\
\hline
\end{tabular}

${ }^{*}$ Muy poco importante (MPI); poco importante (PI); medianamente importante (Mel); importante (I); muy importante (MI). Fuente: elaboración propia.

Tabla 7. Importancia que debe darse a los siguientes aspectos relacionados con el estudiante (\%)

\begin{tabular}{|l|l|l|l|l|l|l|}
\hline \multicolumn{1}{|c|}{ Aspecto } & \multicolumn{3}{c|}{ Valoración* (\%) } \\
\cline { 2 - 6 } & MPI & \multicolumn{1}{|c|}{ PI } & Mel & \multicolumn{1}{|c|}{ I } & MI & \multicolumn{1}{|c|}{ NR } \\
\hline Tener conocimientos disciplinares. & 0,0 & 3,8 & 15,8 & 36,8 & 42,1 & 1,5 \\
\hline Tener un conocimiento general, pero también el detalle de lo que se discute. & 0,8 & 0,8 & 6,8 & 42,1 & 49,6 & 0,0 \\
\hline Tener actitud y motivación hacia las ciencias y sus implicaciones. & 2,3 & 0,0 & 9,0 & 21,1 & 66,9 & 0,8 \\
\hline Saber escuchar al otro en una discusión. & 1,5 & 0,0 & 5,3 & 12,8 & 79,7 & 0,8 \\
\hline Saber identificar en los argumentos los aspectos que generan controversia. & 0,0 & 3,0 & 3,8 & 26,3 & 66,9 & 0,0 \\
\hline
\end{tabular}

* Muy poco importante (MPI); poco importante (PI); medianamente importante (Mel); importante (I); muy importante (MI); no responde (NR). Fuente: elaboración propia.

Tabla 8. Ubicado en la posición del docente, cuál es su percepción acerca de promover la argumentación en clase de ciencias $(\%)$

\begin{tabular}{|l|c|c|c|c|c|c|}
\hline \multirow{2}{*}{ Adjetivo } & \multicolumn{9}{c|}{ Valoración (\%) } \\
\cline { 2 - 9 } & $\mathbf{1}$ & $\mathbf{2}$ & $\mathbf{3}$ & $\mathbf{4}$ & $\mathbf{5}$ & No responde \\
\hline Participativa/Pasiva. & 52,6 & 14,3 & 10,5 & 9,0 & 9,8 & 3,8 \\
\hline Estresante/Relajada. & 8,3 & 5,3 & 21,8 & 20,3 & 37,6 & 6,8 \\
\hline Compleja/Simple. & 8,3 & 15,8 & 36,1 & 17,3 & 14,3 & 8,3 \\
\hline Entretenida/Aburrida. & 63,2 & 12,0 & 10,5 & 3,8 & 4,5 & 6,0 \\
\hline Optimista/Pesimista. & 53,4 & 12,8 & 12,0 & 7,5 & 4,5 & 9,8 \\
\hline Superficial/Profunda. & 6,8 & 3,8 & 10,5 & 28,6 & 44,4 & 6,0 \\
\hline Positiva/Negativa. & 52,6 & 14,3 & 16,5 & 4,5 & 3,8 & 8,3 \\
\hline Teórica/Práctica. & 2,3 & 3,0 & 44,4 & 14,3 & 29,3 & 6,8 \\
\hline Relevante/Secundaria. & 43,6 & 12,0 & 23,3 & 9,8 & 5,3 & 6,0 \\
\hline
\end{tabular}

Fuente: elaboración propia. 
Al igual que en la pregunta anterior, se le solicitó a los estudiantes ubicarse en el rol de docentes de ciencias naturales, para expresar su grado de acuerdo con algunas declaraciones metacognitivas (Tabla 9). Se encontró que al menos el $72,2 \%$ de ellos considera que en general saben lo que se espera lograr cuando se enseña a argumentar en el aula; saben cuándo un estudiante argumenta en clase de ciencias; piensan en lo que necesitan saber antes de iniciar la ejecución de la tarea; tienen un propósito claro para la ejecución de la tarea; analizan la utilidad de las posibles estrategias para promover la argumentación en ciencias; analizan la utilidad, para su desempeño profesional, de emprender este reto e intentar lograrlo; y son conscientes de que el interés que tienen para el desarrollo de la tarea facilita el logro del objetivo propuesto.

Tabla 9. Ubicado en la posición del docente, cuál es su percepción acerca de promover la argumentación en clase de ciencias (\%)

\begin{tabular}{|c|c|c|c|c|c|c|}
\hline \multirow{2}{*}{ Aspecto } & \multicolumn{6}{|c|}{ Valoración* (\%) } \\
\hline & TD & D & $\mathbf{N}$ & A & MA & NS/NR \\
\hline Sé lo necesario sobre argumentación para promoverla en el aula. & 2,3 & 18,8 & 34,6 & 27,8 & 15,8 & 0,8 \\
\hline $\begin{array}{l}\text { Podría explicar con mis palabras lo que es la argumentación en } \\
\text { ciencias. }\end{array}$ & 3,0 & 9,0 & 27,1 & 50,4 & 9,8 & 0,8 \\
\hline $\begin{array}{l}\text { Conozco mis debilidades y fortalezas en la argumentación en } \\
\text { ciencias y su enseñanza en el aula. }\end{array}$ & 2,3 & 9,8 & 23,3 & 43,6 & 18,8 & 2,3 \\
\hline Sé cómo promover la argumentación en el aula. & 0,8 & 6,8 & 33,8 & 43,6 & 12,8 & 2,3 \\
\hline Sé lo que se espera lograr cuando se enseña a argumentar en el aula. & 1,5 & 2,3 & 23,3 & 51,9 & 20,3 & 0,8 \\
\hline Sé cuándo un estudiante argumenta en clase de ciencias. & 0,0 & 1,5 & 18,0 & 60,2 & 19,5 & 0,8 \\
\hline Sé identificar los datos de un argumento. & 1,5 & 8,3 & 26,3 & 48,1 & 15,0 & 0,8 \\
\hline $\begin{array}{l}\text { Sé identificar los fundamentos teóricos que sustentan los } \\
\text { argumentos. }\end{array}$ & 0,0 & 5,3 & 29,3 & 43,6 & 20,3 & 1,5 \\
\hline $\begin{array}{l}\text { Pienso en lo que necesito saber antes de iniciar la ejecución de la } \\
\text { tarea. }\end{array}$ & 1,5 & 3,8 & 9,0 & 42,1 & 42,1 & 1,5 \\
\hline Tengo un propósito claro para la ejecución de la tarea. & 0,8 & 2,3 & 12,0 & 48,1 & 35,3 & 1,5 \\
\hline $\begin{array}{l}\text { Analizo la utilidad de las posibles estrategias para promover la } \\
\text { argumentación en ciencias. }\end{array}$ & 0,8 & 3,0 & 19,5 & 52,6 & 23,3 & 0,8 \\
\hline $\begin{array}{l}\text { Sé cómo abordar los contenidos para facilitar la argumentación en } \\
\text { ciencias. }\end{array}$ & 0,8 & 9,0 & 29,3 & 44,4 & 15,8 & 0,8 \\
\hline $\begin{array}{l}\text { Estoy seguro de lo que creo saber sobre la argumentación en ciencias } \\
\text { y su enseñanza. }\end{array}$ & 0,0 & 15,8 & 29,3 & 45,1 & 9,0 & 0,8 \\
\hline Sé identificar las justificaciones en un argumento. & 0,0 & 9,0 & 24,1 & 53,4 & 12,8 & 0,8 \\
\hline $\begin{array}{l}\text { Pienso que lo que sé sobre argumentación en ciencias debe } \\
\text { promoverse en el aula. }\end{array}$ & 2,3 & 13,5 & 33,1 & 38,3 & 10,5 & 2,3 \\
\hline $\begin{array}{l}\text { Pienso que no tendré dificultades para promover la argumentación } \\
\text { en ciencias en el aula. }\end{array}$ & 2,3 & 26,3 & 33,8 & 30,1 & 7,5 & 0,0 \\
\hline $\begin{array}{l}\text { Analizo la utilidad, para mi desempeño profesional, de emprender } \\
\text { este reto e intentar lograrlo. }\end{array}$ & 0,8 & 3,0 & 14,3 & 47,4 & 33,8 & 0,8 \\
\hline $\begin{array}{l}\text { Soy consciente de que el interés que tengo en el desarrollo de la tarea } \\
\text { facilita el logro del objetivo propuesto. }\end{array}$ & 0,8 & 1,5 & 12,8 & 48,1 & 36,1 & 0,8 \\
\hline
\end{tabular}

* Total desacuerdo (TD); en desacuerdo (D); neutral (N); de acuerdo (A); muy de acuerdo (MA); no sabe/no responde (NS/NR).

Fuente: elaboración propia. 
Así mismo, están en desacuerdo con afirmar no tener dificultades para lograr promover la argumentación en ciencias en el aula o creer que, con lo que saben sobre la argumentación en ciencias y su enseñanza, es suficiente para lograrla en el aula.

La Figura 1 muestra que el 60\% de los estudiantes tienen claridad en dónde están los datos en un fragmento de texto y el 63\% dónde se ubica la justificación de este, pero no dónde está la tesis central. El semestre o el género no mostraron dependencia con ninguna de las tres preguntas realizadas en este item (valores $\mathrm{P}=0,115$ o mayores).

\section{Discusión de resultados}

Un apoyo importante para la discusión, que dio mayor alcance a los resultados, fue ubicarnos en las columnas 1 y 2 o 4 y 5 de las valoraciones (Tablas 8 y 9), esto porque la neutral nos ubica en una situación de no concreción de la perspectiva del estudiante frente a la proposición planteada.

El primer aspecto indagado correspondió a lo que significa argumentar en ciencias. Para la primera afirmación, correspondiente a una perspectiva informal de la argumentación, el resultado es del $66,2 \%$; para la segunda afirmación, correspondiente a una perspectiva epistémica (como artefacto epistémico), el resultado es de $84,2 \%$; para la tercera afirmación, relacionada con una perspectiva pragmática, el resultado es de $72,9 \%$; y, por último, para la perspectiva lógico formal, el resultado es de $73,7 \%$. Estos resultados muestran claramente la diversidad conceptual de nuestros estudiantes y posibles limitaciones que deben intervenirse.

Una perspectiva lógico formal de la argumentación tendría como posibles dificultades que, al ser contextos de debate artificiales, es difícil relacionar elementos de orden social y cultural con los procesos argumentativos (Cano, 2010). La perspectiva pragmática, a pesar de sustentarse en razonamientos informales y contextuales, sigue conservando el estatismo de la lógica formal, generado por el carácter unidireccional del proceso argumentativo que reconoce, más allá de la interacción social, las estrategias del orador para persuadir al auditorio (Cano, 2010). La perspectiva epistémica (Knuuttila, 2005; Leitão, 2000; Ruiz y Ocampo, 2019) tiene como elemento relevante asumir la argumentación como proceso dialógico,

Figura 1. Justificación, tesis central y datos de un fragmento de texto

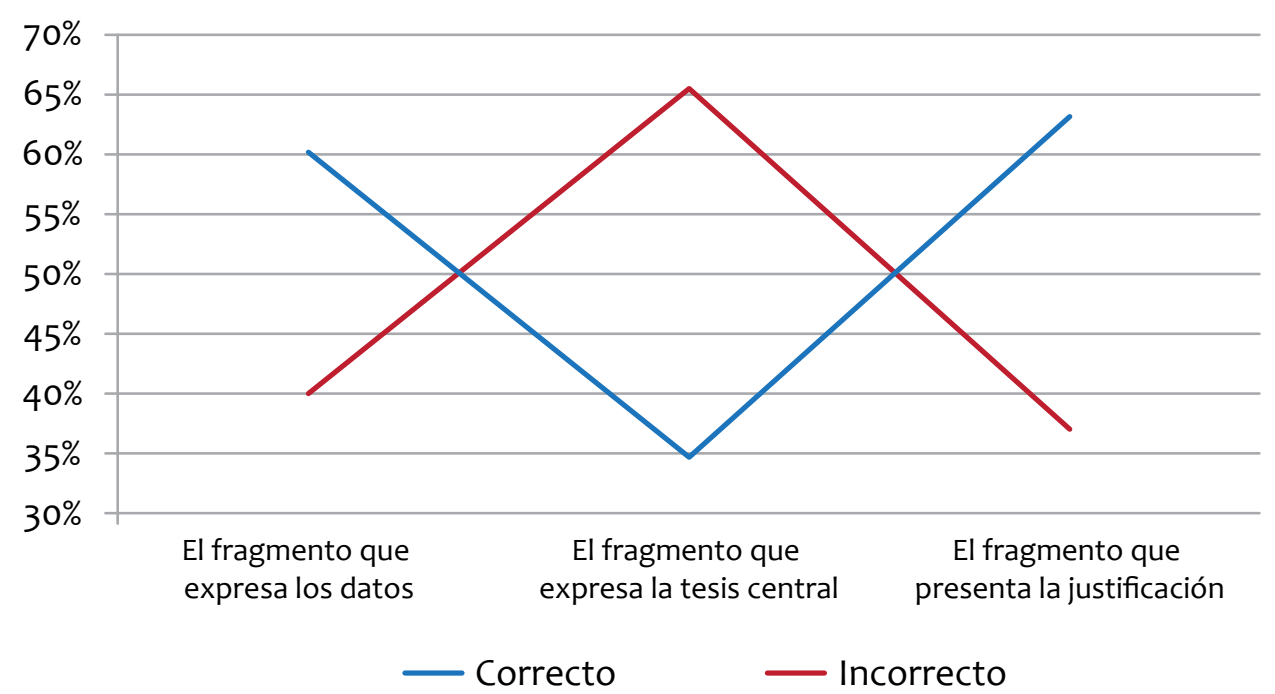

Fuente: elaboración propia. 
dialéctico y situado; sin embargo, presenta limitaciones que dificultan su incorporación, tales como: incipientes estudios que relacionen, de un lado, aspectos emocionales con procesos argumentativos $y$, de otro lado, el componente reflexivo y consciente de los sujetos, como elemento mediador y cualificador de los propios argumentos.

Para el objetivo de la argumentación, al igual que lo realizado para el concepto, al sumar las dos últimas columnas se observa que las afirmaciones con mayor porcentaje de alta valoración (4 y 5) son dos intencionalidades totalmente opuestas: la argumentación para la reestructuración del conocimiento (articulada a la perspectiva epistémica) y la argumentación para el convencimiento (vinculada a la perspectiva formal), con valores del $60,2 \%$ y $75,9 \%$, respectivamente. La primera está apoyada en el desarrollo de procesos comunicacionales que incorporan el reconocimiento de los aspectos socioculturales de los sujetos y sus saberes (Jiménez, 1998; Ruiz, Tamayo y Márquez, 2015) y es, desde nuestra perspectiva, la intencionalidad que ayudaría a la reconstrucción del conocimiento en el aula. La segunda, apoyada en la estructura rigurosa de los argumentos, como elemento central que garantice el convencimiento, desconoce los elementos contextuales de los escenarios de debate.

Sobre la pregunta por las actividades que más se proponen en sus aulas de clase, se tienen dos datos representativos, al sumar las valoraciones más altas (columnas 4 y 5 de la Tabla 4): la resolución de ejercicios matemáticos, físicos o químicos, con $90,7 \%$, y las pruebas escritas, con $78,2 \%$. Datos que van en contravía de lo que proponen los mismos estudiantes como actividades necesarias para desarrollar la argumentación en ciencias: análisis de casos $(80,4 \%)$, debates $(75,2 \%)$, resolución de controversias sociocientíficas $(69,2 \%)$ y trabajos grupales $(68,4 \%)$, actividades que aportan al desarrollo de la argumentación, por varias razones. La primera, porque se acepta la presencia de un auditorio, es decir, la interacción intersubjetiva, que no necesariamente implica la renuncia a las propias posiciones ( $\mathrm{Si}$ - monneaux, 2001; Kuhn, 1993, Leitão, 2000); más que eso, significa la posibilidad que dan las disonancias de comprender en profundidad los temas que se debaten. La segunda, porque se reconoce el trabajo en pequeños grupos como una estrategia que transforma el papel del docente como portavoz de la ciencia, hacia una perspectiva del docente como mediador y facilitador de la pluralidad de puntos de vista en el aula (Osborne, 2009).

En relación con los criterios asignados al docente, al estudiante y al contenido, los resultados muestran aspectos interesantes, más allá de la independencia estadística entre el semestre en el cual se ubican los estudiantes y el género. El primero, la importancia del contenido disciplinar para poder desarrollar argumentos de calidad. Con este criterio, asignado tanto al docente como al estudiante, se ratifica la perspectiva propuesta por Kuhn (1993), para quien el aprendizaje es un proceso argumentativo, donde el aspecto disciplinar es fundamental para la participación razonada en los diálogos sobre los temas que se debaten en el aula. El segundo aspecto tiene que ver con la contextualización de los contenidos. Pretender desarrollar procesos argumentativos apoyados en un contenido disciplinar claro, coherente y preciso exige del docente diseñar escenarios de enseñanza y aprendizaje y, en nuestro caso, escenarios de discusión, que permitan a los estudiantes exponer lo que piensan y saben, de una manera espontánea, tranquila, y con la idea de que serán objeto de discusión en dichos encuentros. El último aspecto está relacionado con el reconocimiento de la parte emocional y afectiva, como dispositivos que limitan o potencian el desarrollo de los procesos argumentativos. Varios autores plantean que las emociones deben formar parte explícita de los estudios relacionados con los procesos argumentativos (Habermas, 1987; Sotomayor, 2017; Plantin, 2011; Santibáñez, 2014). Como bien apunta Molina, "negar la base emocional no solo en la argumentación propiamente dicha sino también en la elección de los temas, de los objetivos y en el alcance de los acuerdos logrados, es negar que los niños -y cualquier sujeto involucrado en los debates- sean 
capaces de mantener discusiones críticas con sus pares y que esas discusiones sean relevantes y necesarias para ellos" (2012, p. 9). Argumentar promueve en los estudiantes actitudes y valores: "al aprender a argumentar también se potencian otras competencias de orden cognitivo como la crítica, la reflexión y también actitudes y valores, indispensables para que el sujeto, como ciudadano activo, tome decisiones e intervenga su entorno inmediato" (Ruiz, 2012, p. 23).

Todo lo anterior exige, como lo plantean Zabala y Arnau (2007), que los temas o fenómenos que se discuten en el aula signifiquen algo para la vida de los estudiantes, que sean temas cercanos a las vivencias e intereses de los estudiantes (Couso et al. 2005; Jiménez, 1998).

En relación con la percepción del estudiante del reto de promover la argumentación en el aula, hay tres adjetivos que, al sumarse las columnas 1 y 2 ó 4 y 5 de la Tabla 8, sobresalen en las valoraciones más altas de las clases como relajadas (57,9\%), profundas (73\%) y prácticas (43,6\%). En la primera valoración, se destaca, posiblemente, un sentimiento de confianza frente a este reto (Sutton y Wheatley, 2003; Vloet, 2009), aspecto positivo que sin duda facilita el desempeño y el desarrollo de prácticas eficientes y eficaces. Los otros dos adjetivos que obtuvieron las calificaciones más altas: profunda y práctica, evidencian la exigente tarea para el maestro de programar rigurosamente escenarios donde la argumentación se proyecte como actividad indispensable para "resolver" problemas y evitar quedarse solo en el plano discursivo y teórico; importante, claro está, pero insuficiente, si se trata de aportar al desarrollo de ciudadanos que intervengan en el cambio social.

Para la pregunta que indagó sobre aspectos metacognitivos, en la literatura referida a este tema se plantea que, si pretendemos desarrollar habilidades de orden superior, como la explicación, la inferencia, la reflexión o la argumentación, es necesario incorporar procesos de orden metacognitivo, tanto en docentes como en estudiantes (Schraw y Denni- son, 1994; Zimmerman y Moylan, 2009; Panadero y Alonso, 2014). En este sentido, la pregunta hecha a los estudiantes se ubicó en los denominados juicios metacognitivos (Nelson y Narens, 1990; Dunlosky et al., 2013). Para Winne: "ser capaz de hacer juicios metacognitivos precisos a lo largo del proceso de aprendizaje es necesario para tomar sabias decisiones de estudio sobre la ejecución y planificación de tareas" (1995, citado por Hadwin y Webster, 2013, p. 38).

En los resultados obtenidos tras la aplicación del cuestionario, al sumar los porcentajes de las columnas 4 y 5 (de acuerdo y totalmente de acuerdo), se pueden evidenciar, al menos, tres aspectos importantes. El primero, los juicios de confianza de los estudiantes (representados en las afirmaciones que se les propusieron) apoyan la idea de que enseñar a argumentar en el aula exige tener conciencia del propósito de la tarea y los saberes requeridos para alcanzarlo. Lo anterior se puede evidenciar en los porcentajes obtenidos para afirmaciones como "sé lo que se espera lograr cuando se enseña a argumentar en el aula", con $72,2 \%$ de respuestas (que valoran estar de acuerdo y totalmente de acuerdo); "tengo un propósito claro para la ejecución de la tarea", con 83,4\%; "pienso en lo que necesito saber antes de iniciar la ejecución de la tarea", con $84,2 \%$, y "soy consciente de que el interés que tengo para el desarrollo de la tarea facilita el logro del objetivo propuesto", con $84,2 \%$.

El segundo aspecto lo encontramos cuando los estudiantes manifiestan estar de acuerdo y totalmente de acuerdo en porcentajes inferiores al 50\% para afirmaciones como: "sé lo necesario sobre la argumentación para promoverla en el aula", con 43,6\%; "pienso que lo que sé sobre la argumentación en ciencias es la perspectiva que debe promoverse en el aula", con 48,8\%; "creo que con lo que sé sobre la argumentación en ciencias y su enseñanza es suficiente para lograrla en el aula", con 30,9\%; "sé identificar la tesis central en un argumento", con 42,1\%; "pienso que no tendré dificultades para pro- 
mover la argumentación en ciencias en el aula", con $37,6 \%$. Si notamos, las cuatro primeras afirmaciones hacen referencia al conocimiento que debería tener el estudiante sobre la argumentación; en ellas, es evidente la inseguridad sobre lo que supone argumentar en ciencias y la confusión sobre lo que es la argumentación y su propósito (resultado coherente con lo identificado en las dos primeras preguntas discutidas en esta investigación). Ahora bien, la última afirmación, que tiene que ver con la reflexión sobre posibles dificultades: el nivel de acuerdo y de estar totalmente de acuerdo, presenta uno de los resultados más bajos (37,6\%), como evidencia mucho más clara de las falencias que reconocen tener los futuros docentes y de la posibilidad de intervenirlas que tiene el programa de licenciatura en Biología y Química de la Universidad de Caldas.

La última actividad planteada en el cuestionario se relacionó con la acreditación (ver nota a pie de página número 2), donde los estudiantes debían identificar los datos, la justificación y la tesis central. Los resultados ratifican aún más las dificultades de los estudiantes en los procesos argumentativos. Si bien los porcentajes correctos para datos y justificaciones superaron el $60 \%$ de asertividad -mientras se esperaría que fueran mucho más altos-, para el caso de la identificación de la tesis el porcentaje fue inferior a $40 \%$. Recordemos que un proceso argumentativo en el aula de ciencias requiere que los estudiantes asuman una posición clara o logren identificar perspectivas conceptuales o teóricas sobre el tema o fenómeno que se discute. Si esto no está claro para los estudiantes, posiblemente tendrán dificultades para la presentación de pruebas científicas que permitan apoyar sus perspectivas $y$, de igual manera, justificar por qué sus pruebas pueden apoyar sus perspectivas o la toma de postura sobre lo discutido.

\section{Conclusiones}

A la luz de la discusión realizada acerca de los resultados obtenidos, se ratifica la necesidad de in- tervenir y proponer la argumentación como un contenido explícito en la formación de licenciados en Biología y Química, toda vez que los acercamientos manifiestos en los futuros docentes hacia posturas teóricas sobre lo que supone argumentar en ciencias, desde la lógica formal, la pragmática y la perspectiva epistémica, evidencian la necesidad de intervenir su pensamiento. Hacerlo permitirá comprender el alcance que tiene cada una de estas perspectivas, en función de procesos de enseñanza y aprendizaje, proyectados al desarrollo, entre otras cosas, de aprendizajes profundos, de habilidades de pensamiento de orden superior, de actitudes y valores hacia la ciencia y hacia, y también de comprensiones mucho más profundas sobe la ciencia y de la ciencia.

El uso de actividades mayoritariamente sustentadas en el desarrollo de ejercicios matemáticos, físicos o químicos, que restringen el desarrollo de aprendizajes en profundidad y procesos argumentativos propios del pensador crítico, limita el desarrollo de procesos argumentativos. Una posible explicación podría encontrarse, quizás, en la formación académica y profesional de quienes tienen bajo su responsabilidad la orientación de las diferentes asignaturas en la licenciatura, pues seguramente fueron formados en estructuras curriculares rígidas, en las cuales los procesos de debate fueron escasos o nulos. Frente a esto, está la invitación a que en la licenciatura se brinden espacios donde revisar el alcance que tiene para los procesos de enseñanza y aprendizaje la incorporación de debates, estudios de caso y trabajos grupales, reconocidos por los futuros docentes como ejes indispensables para desarrollar la argumentación en ciencias.

Finalmente, la poca claridad para asumir la argumentación como posibilidad de aprender y coconstruir conocimiento escolar confirma la necesidad de incorporar en los procesos de formación de los futuros docentes la crítica, el razonamiento, la actividad grupal y los juicios metacognitivos, para que el futuro docente cualifique sus propios desempeños. 


\section{Agradecimientos}

A la Universidad de Caldas y, en ella, al programa y los estudiantes de la Licenciatura en Ciencias Natu- rales. Al Programa Reconstrucción del Tejido Social en Zonas de Postconflicto en Colombia, con código de la Vicerrectoría de Investigaciones y Postgrados 2012917.

\section{Referencias}

Archila, P. A. (2014). La argumentación en la formación de profesores de química: relaciones con la comprensión de la historia de la química. Revista Científica, 18, 50-66. Recuperado de: https://revistas.udistrital.edu.co/ index.php/revcie/article/view/5561/7075

Archila, P. A. (2016). ¿Cómo formar profesores de ciencias que promuevan la argumentación?: Lo que sugieren avances actuales de investigación. Profesorado. Revista de Curriculum y Formación de Profesorado, 20(3), 399-432. Recuperado de: https://www.redalyc.org/pdf/567/56749100oo9.pdf

Cano, M. I. (2010). Argumentació i construcció del coneixement: Estratègies argumentatives dels estudiants universitaris en situació de debat. [tesis doctoral] Universitat Ramon Llull, Barcelona.

Cochran-Smith, M. y Lytle, S. (2003). Más allá de la certidumbre: adoptar una actitud indagadora sobre la práctica. En A. Lieberman y L. Millar (eds.), La indagación como base de la formación del profesorado y la mejora de la educación (pp. 65-80). Barcelona: Octaedro. Recuperado de: https://www.researchgate.net/publication/283523147_Mas_alla_de_la_certidumbre_adoptar_una_actitud_indagadora_sobre_la_practica

Couso, D., Cadillo, E., Perafán, G.y Adúriz-Bravo, A. (2005). Unidades didácticas en ciencias y matemáticas. Bogotá: Didácticas Magisterio.

Cutrera, G. y Stipcich, S. (2015). La explicación en el aula de ciencias: cómo enseñamos a explicar. Un estudio centrado en el discurso de un docente en formación. En F. Santillán Campos (coord.), Investigación educativa en Latinoamérica (pp. 199-208). México: Centro de Estudios e Investigaciones para el Desarrollo Docente. Recuperado de: https://dialnet.unirioja.es/servlet/articulo?codigo $=5434993$

Dunlosky, J., Rawson, K. A., Marsh, E. J., Nathan, M. J., y Willingham, D. T. (2013). Improving students' learning with effective learning techniques: Promising directions from cognitive and educational psychology. Psychological Science in the Public Interest, 14(1), 4-58. DOI: http://doi.org/10.1177/1529100612453266

Echeverría, P. (2010). El papel de la docencia universitaria en la formación inicial de profesores. Calidad en la Educación, 32, 150-165. Recuperado de: https://doi.org/10.31619/caledu.n32.154

Habermas, J. (1987). Teoría de la acción comunicativa (vol. I). Madrid: Taurus.

Hadwin, A. F.y Webster, E. A. (2013). Calibration in goal setting: Examining the nature of judgments of confidence. Learning y Instruction, 24, 37-47. DOI: https://doi.org/10.1016/j.learninstruc.2012.10.001 
Hernández Sampieri, R., Fernández Collado, C., Baptista Lucio, M. (2010). Metodología de la investigación (5 ed.). México: McGraw-Hill.

Jiménez-Aleixandre, M. P. (1998). Diseño curricular: indagación y razonamiento con el lenguaje de las ciencias. Enseñanza de las Ciencias, 16(2), 203-216. Recuperado de: https://www.researchgate.net/publication/28051983_Diseno_curricular_indagacion_con_el_lenguaje_de_las_ciencias

Knuuttila, T. (2005). Models, representation, and mediation. Philosophy of Science, 72, 1261-1271. Recuperado de: https://doi.org/10.1086/508124

Kuhn, D. (1993). Science as argument: Implications for teaching and learning scientific thinking. Science Education, 77, 319-337. DOI: https://doi.org/10.1002/sce.3730770306

Landazábal, D. y Gamboa, M. (2018). El proceso de argumentación en la formación inicial de docentes: una experiencia mediada por Dígalo y Simas. Bogotá: Universidad Distrital Francisco José de Caldas.

Leitão, S. (2000). The potential of argument in knowledge building. Human Development, 6, 332-360. DOI: https://doi.org/10.1159/000022695

Lourenço, A., Abib, M. y Murillo, F. (2016). Aprendendo a ensinar e a argumentar: saberes de argumentação docente na formação de futuros professores de química. Revista Brasileira de Pesquisa em Educação em Ciências, 16(2), 295-316. Recuperado de: https://periodicos.ufmg.br/index.php/rbpec/article/view/4376

Mellado, V. (2001). ¿Por qué a los profesores de ciencias nos cuesta tanto cambiar nuestras concepciones y modelos didácticos? Revista Interuniversitaria de Formación del Profesorado, 40, 17-30. Recuperado de: https:// dialnet.unirioja.es/servlet/articulo?codigo=118089

Mellado, V. (2010). Formación del profesorado de ciencias y buenas prácticas: el lugar de la innovación y la investigación didáctica. En A. Caamaño, (coord.), Física y química. Investigación, innovación y buenas prácticas (pp. 11-30). Barcelona: Grao. Recuperado de: https://www.researchgate.net/publication/282815154_Formacion_del_profesorado_y_buenas_practicas_el_lugar_de_la_innovacion_y_la_investigacion_educativa

Molina, M. (2012). Estudio de las emociones en la argumentación infantil. RILL Nueva Época, Prácticas Discursivas a Través de las Disciplinas, 17(1-2) 1-11. Recuperado de: https://www.aacademica.org/maria.elena.molina/28.pdf

Muller, N., y Perret-Clermont, A. N. (2009). Argumentation and education: Theoretical foundations and practices. Nueva York: Springer.

Nelson, T. O. y Narens, L. (1990). Metamemory: A theoretical framework and some new findings. En G. H. Bower (ed.), Thepsychology offearning und motivation (vol. 26, pp. 125-173). San Diego: Academic. DOI: https://doi. org/10.1016/S0079-7421(08)60053-5

Osborne, J. (2009). Hacia una pedagogía más social en la educación científica: el papel de la argumentación. Educación Química, 20(2), 145-154. DOI: https://doi.org/10.1016/S0187-893X(18)30022-3 
Panadero, E. y Alonso-Tapia, J. (2014). How do students self-regulate? Review of Zimmerman's cyclical model of self-regulated learning. Anales de Psicología, 30(2), 450-462. DOl: https://doi.org/10.6018/analesps.30.2.167221

Paglieri, F. y Castelfranchi, C. (2010). Why arguing? Towards a costs-benefits analysis of argumentation. Argument y Computation, 1, 71-91. DOI: https://doi.org/10.1080/19462160903494584

Plantin, C. (2011). Les bonnes raisons des émotions. Principes et méthode pour l'analyse du discours émotionné. Berna: Peter Lang.

Ruiz, F. J. (2012). Caracterización y evolución de los modelos de enseñanza de la argumentación en clase de ciencias en la educación primaria. Bellatera, Barcelona: Universidad Autónoma de Barcelona. Recuperado de: https://www.raco.cat/index.php/Ensenanza/article/view/285759

Ruiz, F. (2016). La reflexión sobre la práctica: una herramienta indispensable para movilizar el pensamiento del docente en formación. En M. González, A. Barats y A. Brandi (eds.), Actas del IV Congreso de Docentes de la Naturaleza (pp. 453-460). Madrid: Santillana. Recuperado de: https://www.researchgate.net/publication/316659731_la_reflexion_sobre_la_Practica_una_herramienta_indisPensable_Para_movilizar_el_Pensamiento_del_docente_en_formacion\#fullTextFileContent

Ruiz Ortega, F. J., Tamayo Alzate, O. E. y Márquez Bargalló, C. (2015). A model for teaching argumentation in science class. Educação e Pesquisa, 41(3), 629-646. DOI: https://doi.org/10.159o/S1517-9702201507129480

Ruiz, F., Márquez, C., Badillo, E. y Rodríguez, M. (2018). Desarrollo de la mirada profesional sobre la argumentación científica en el aula de secundaria. Revista Complutense de Educación, 29(2), 35-52. DOI: https://doi. org/10.5209/RCED.53452

Ruiz F. y Ocampo, L. (2019). Relaciones de cooperación y especialización entre la argumentación y múltiples lenguajes en la clase de ciencias. Didacticae: Revista de Investigación en Didácticas Especificas, 5, 57-72. DOI: https://doi.org/10.1344/did.2019.5.57-72

Sanmartí, N. (2019). La enseñanza y el aprendizaje de la biología mediante el trabajo por proyectos. [Conferencia] $X$ Encuentro Nacional de Experiencias en Enseñanza de la Biología y la Educación Ambiental, $V$ Congreso Nacional de Investigación en Enseñanza de la Biología, Bogotá, Colombia.

Santibáñez, C. (2014). ¿Para qué sirve argumentar?: Problematizando teórica y empíricamente el valor y la función de la argumentación. Círculo de Lingüística Aplicada a la Comunicación, 58, 163-205. DOI: https://doi. org/10.5209/rev_CLAC.2014.v58.45474

Schraw, G. y Dennison, R. S. (1994). Assessing metacognitive awareness. Contemporary Educational Psychology, 19, 460-475. DOI: https://doi.org/10.1006/ceps.1994.1033

Simonneaux, L. (2001). Role-Play or debate to promote students' argumentation and justification on an issue in animal transgenesis. International Journal of Science Education, 23, 903-927. 
Sotomayor, J. E. (2017). Emoción, racionalidad y argumentación en la decisión judicial. Derecho PUCP, 79, 151-190. DOI: https://doi.org/10.1880o/derechopucp.201702.008

Sprent, P. y Smeeton, N. C. (2007). Applied nonparametric statistical methods (4 ed.). Boca Ratón, Fl.: Chapman y Hall/CRC. DOI: https://doi.org/10.1007/s11336-010-9166-4

Stipcich, S. (2005). La argumentación en las clases de física. En S. Stipcich, Las disciplinas, las áreas: problemáticas de su enseñanza (pp. 152-156). Argentina: uncPBA. (Universidad Nacional de Centro de Provincia de Buenos Aires).

Stipcich, M. S., Islas, M. y Domínguez, A. (2006). El lugar de la argumentación en la formación de profesores de ciencias. Revista Chilena de Educación Científica, 6(1), 67-74.

Sutton, R. E.yWheatley, K.F. (2003). Teachers'emotions and teaching: Areview of the literatureand directions forfuture research. Educational Psychology Review, 15(4), 327-358. Recuperado de: https://doi.org/10.1023/A:1026131715856

Tezanos, A. (2013). La formación de educadores y la calidad de la educación. Revista Educación y Pedagogía, 14-15, 36-58. Recuperado de: https://revistas.udea.edu.co/index.php/revistaeyp/article/view/5579

Vianna, D. y Carvalho, A. M. (2000). Formação permanente: a necessidade da interação entre a ciência dos cientistas e a ciência da sala de aula. Ciência y Educação, 6(1), 31-42. Recuperado de: https://doi.org/10.1590/ S1516-73132000000100004

Vloet, K. (2009). Career learning and teachers' professional identity: Narratives in dialogue. En M. Kuijpers y F. Meijers (eds.), Career learning. Research and practice in education (pp. 69-84). Hertogenbosch, Holanda: Euro-Guidance. Recuperado de: https://www.researchgate.net/publication/255580655_Career_learning_ and_teachers\%27_professional_identity_Narratives_in_dialogue

Zabala, A. y Arnau, L. (2007). 11 ideas clave. Cómo aprender y enseñar competencias. Barcelona: Graó.

Zimmerman, B. J. y Moylan, A. R. (2009). Self-regulation: Where metacognition and motivation intersect. En D. J. Hacker, J. Dunlosky y A. C. Graesser (eds.), Handbook of metacognition in education (pp. 299-315). Nueva York: Routledge. Recuperado de: https://ssrlsite.files.wordpress.com/2017/11/routledgehandbooks9780203876428-chapter16-zimmerman.pdf

Zohar, A. (2007). Science teacher education and professional development. En S. Erduran and M. P. JiménezAleixandre (eds.), Argumentation in science education: Perspectives from class- room-based research (pp. 245-268). Nueva York: Springer. https://doi.org/10.1007/978-1-4020-6670-2_12 